\title{
Speckle Reduction of Synthetic Aperture Radar Images using Median Filter and Savitzky-Golay Filter
}

\author{
Ruchita Gir \\ M. Tech Student \\ NIIST, Bhopal \\ Madhya Pradesh, India
}

\author{
Lalit Jain \\ Assistant Professor \\ NIIST, Bhopal \\ Madhya Pradesh, India
}

\author{
Rajesh Rai \\ Professor \\ NIRT, Bhopal \\ Madhya Pradesh, India
}

\begin{abstract}
SAR images are corrupted by speckle noise which is based on multiplicative noise or Reyleigh noise. The speckle degrades the quality of image and makes interpretations, analysis and classifications of SAR images harder. Therefore some speckle mitigation is necessary prior to the processing of SAR images. In this paper a new method is proposed for despeckling of SAR images in which Savitzky-Golay filter and median filter are used for denoising of the synthetic aperature radar (SAR) image. After obtaining filtered image they are decomposed by the use of undecimated wavelet transform. The speckled input image is also decomposed using undecimated wavelet transform. Then image segmentation is done by the use of brute force thresholding wavelet based algorithm in which each pixel of the entire decomposed image is compared and the maximum value of threshold image pixel is replaced in every iteration of image processing. Lastly enhanced directional smoothing of the image is done to obtain a despeckled image
\end{abstract}

\section{General Terms}

Undecimated Wavelet Transform, SAR, Savitzky-Golay filter, median filter, direction dependent mask, Directional Smoothing.

\section{INTRODUCTION}

Synthetic aperture radar (SAR) imaging, due to its powerful imaging capability in all weather conditions, day and night, sunny and cloudy, has become more and more popular in our daily lives and in military tasks. But SAR images are corrupted by speckle noise due to random interference of electromagnetic signals. The speckle degrades the quality of the images and makes interpretations, analysis and classifications of SAR images harder. Thus speckle noise should be removed before applying any image processing techniques. Speckle reduction usually consists of three stages. First stage is to transform the noisy image to a new space (frequency domain). Second stage is the manipulation of coefficients. Third is to transform the resultant coefficients back to the original space (spatial domain)[1].

In wavelet sub bands noise is present in small coefficients and important feature details are present in large coefficients. If removal of small coefficient is done, we can get a noise free image. Therefore in recent years wavelet based speckle reduction is becoming a field of interest among researchers for multi resolution decomposition and analysis of image.

Many algorithms have been developed for de-speckling, including the Lee filter [2], the Frost filter [3], the Gamma MAP filter [4], and their variations [5]. These standard filters usually perform well in despeckling; however, they typically exhibit limitations in preserving sharp features and/or details of the original image [6]. Thus to preserve edges enhanced directional smoothing is applied.

Earlier for reduction of speckle researchers use discrete wavelet transformation [1][4][5][7][8][9].But drawback of DWT is that it is translation variant[10]. Some important coefficients can be lost during transformation from original signal to sub bands. Thus to save coefficients undecimated wavelet transform (UDWT) is used. Basic idea of undecimated wavelet transform is to fill in the gaps caused by decimation step in DWT. UDWT leads to an over-determined and redundant representation of the original data. Whether discrete or undecimated wavelet is used, we need to take care for selecting optimal thresholding.

Many thresholding techniques are used by researchers such as hard, soft, VisuShrink, SureShrink, OracleShrink, NormalShrink, BayesShrink, Thresholding Neural Network (TNN) etc. [11]-[15].But there are some draw backs of using those thresholds as shown in [16]. Thus we are using brute force thresholding which gives best results as compared to above threshold techniques.

\section{BASIC PRINCIPLES OF SPECKLE THEORY AND FILTERING TECHNIQUE}

\subsection{Speckle Model}

A realistic model for explaining a SAR image to simply measuring a patch of homogenous area in SAR image can be expressed as

$$
I(x, y)=R(x, y) . S(x, y)
$$

Where $(\mathrm{x}, \mathrm{y})$ are the spatial range and azimuth co-ordinates of the resolution

$\mathrm{I}(\mathrm{x}, \mathrm{y})$ is the intensity of SAR image i.e. degraded pixel of observed image

$\mathrm{R}(\mathrm{x}, \mathrm{y})$ is the random process of unspeckled radiance i.e. noiseless image pixel

$\mathrm{S}(\mathrm{x}, \mathrm{y})$ is model as speckle noise having stationary random process with unit mean and variance proportional to effective number of looks $\mathrm{N}$.

\subsection{Speckle Filtering Techniques}

The speckle filtering techniques can be classified into two categories i.e. pre-processing and post processing. In this paper both type of filtering technique is used. For preprocess filtering technique median filter and Savitzky-Golay filter is used whereas for post processing enhanced directional smoothening is done to the despeckled image. 


\subsubsection{Preprocess Filtering}

Median filter preserve discontinuities in a step function and smooth a few pixels whose values differ significantly from the surrounding, without affecting the other pixels. Median of a discrete sequence of size $\mathrm{N}$ (odd) is the member of the sequence for which $(\mathrm{N}-1) / 2$ elements are smaller or equal in value and (N-1)/2 are equal or larger in value[19]

Savitzky-Golay smoothing filters are used to smooth out a noisy signal whose frequency span (without noise) is large. This filter perform much better than standard averaging FIR filters, which tend to filter out a significant portion of the signal's high frequency content along with the noise. Savitzky-Golay filters are more effective at preserving the pertinent high frequency components of the signal. SavitzkyGolay filter minimize the least-squares error by fitting a polynomial to frames of noisy data. Least squares smoothing reduces noise while maintaining the height and shape of waveform peaks [17]

\subsubsection{Postprocess Filtering}

To protect the edges from blurring we apply enhanced directional smoothing to the processed despeckled image. Directional smoothing chooses neighbourhood with smallest variance and replace pixel value with the average of that neighbourhood. The EDS filter has a speckle reduction approach that performs spatial filtering in a square-moving window know as kernel. The EDS filtering is based on the statistical relationship between the central pixel and its surround-ding pixels. EDS performs the filtering based on either local statistical data given in the filter window to determine the noise variance within the filter window, or estimating the local noise variance using the effective equivalent number of looks (ENL) of a SAR image. The estimated noise variance is then used to determine the amount of smoothing needed for each speckle image. The noise variance determined from the local filter window is more applicable if the intensity of an area is constant or flat while ENL is suitable if there are difficulties determining if an area of the image is flat[18]

\subsection{Proposed Algorithm}

In proposed algorithm the speckled image is decomposed into wavelet sub bands i.e. horizontal, vertical, diagonal details of image. Maximum and minimum value of sub band coefficient through loop is found. Desired algorithm is executed and results are saved in array for each loop. When loop is completed the optimal threshold is selected which gives the best result.

The steps for the algorithm are as follows:

Assuming A as the original speckled SAR image. B is the Savitzky Golay filtered SAR image of A and C is the median filtered SAR image of A

Decompose the images $\mathrm{A}, \mathrm{B}$ and $\mathrm{C}$ into wavelet sub bands $S(\epsilon)_{i, A}, S(\epsilon)_{i, B}$ and $S(\epsilon)_{i, C}$ using undecimated wavelet transformation. Where (i) is decomposition level and $(\epsilon)$ denotes the detail sub bands such as horizontal detail, vertical detail and diagonal detail sub bands respectively. Decomposition up to level 3 is enough.

Process each level of detail images of A by the following steps
1) Find minimum $(\min )$ and maximum $(\max )$ value of coefficients of each detail sub band at each level.

2) Loop (threshold $=\min$ to $\max$ ) for each detail sub band at each level.

3) Determine whether each pixel of $S(\epsilon)_{i, A}$ is High level or Low level. If (pixel < threshold) then it is low level otherwise pixel is high level

4) If pixel is low level then replace the value of pixel of $S(\epsilon)_{i, A}$ by corresponding pixel value of $S(\epsilon)_{i, C}$

5) To determine whether there exists edges around the pixels that are classified as high level pixels, a direction dependent mask is used. If the dark portion of mask or window contains at least one high level pixel, then keep the original value of pixel intact else replace the value of pixel of $S(\epsilon)_{i, A}$ by corresponding pixel value of $S(\epsilon)_{i, B}$

6) Apply the directional smoothing to each detail sub band at each level $S(\epsilon)_{i, A}$

7) Reconstruct an image from the processed sub bands of $S(\epsilon)_{i, A}$ by using inverse undecimated wavelet transformation.

8) Save the results of each iteration in an array such as $\mathrm{F}=[$ threshold, result $]$.

9) Return to step 2 until loop completed. Select the threshold value that gives best result and use that threshold for classification of high level pixels and low level pixels.

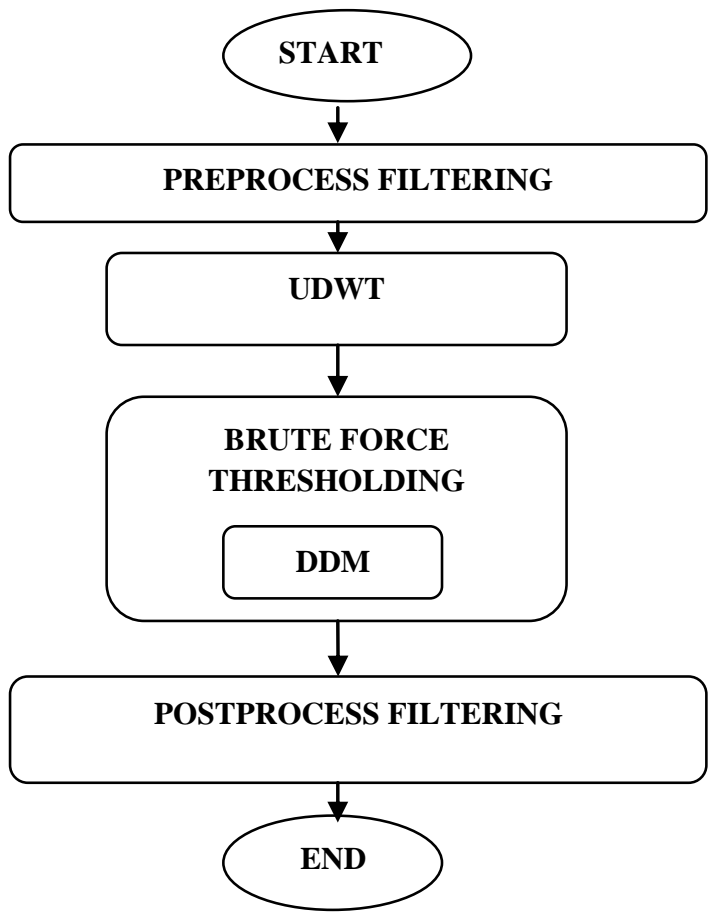

Fig 1. Flow chart 


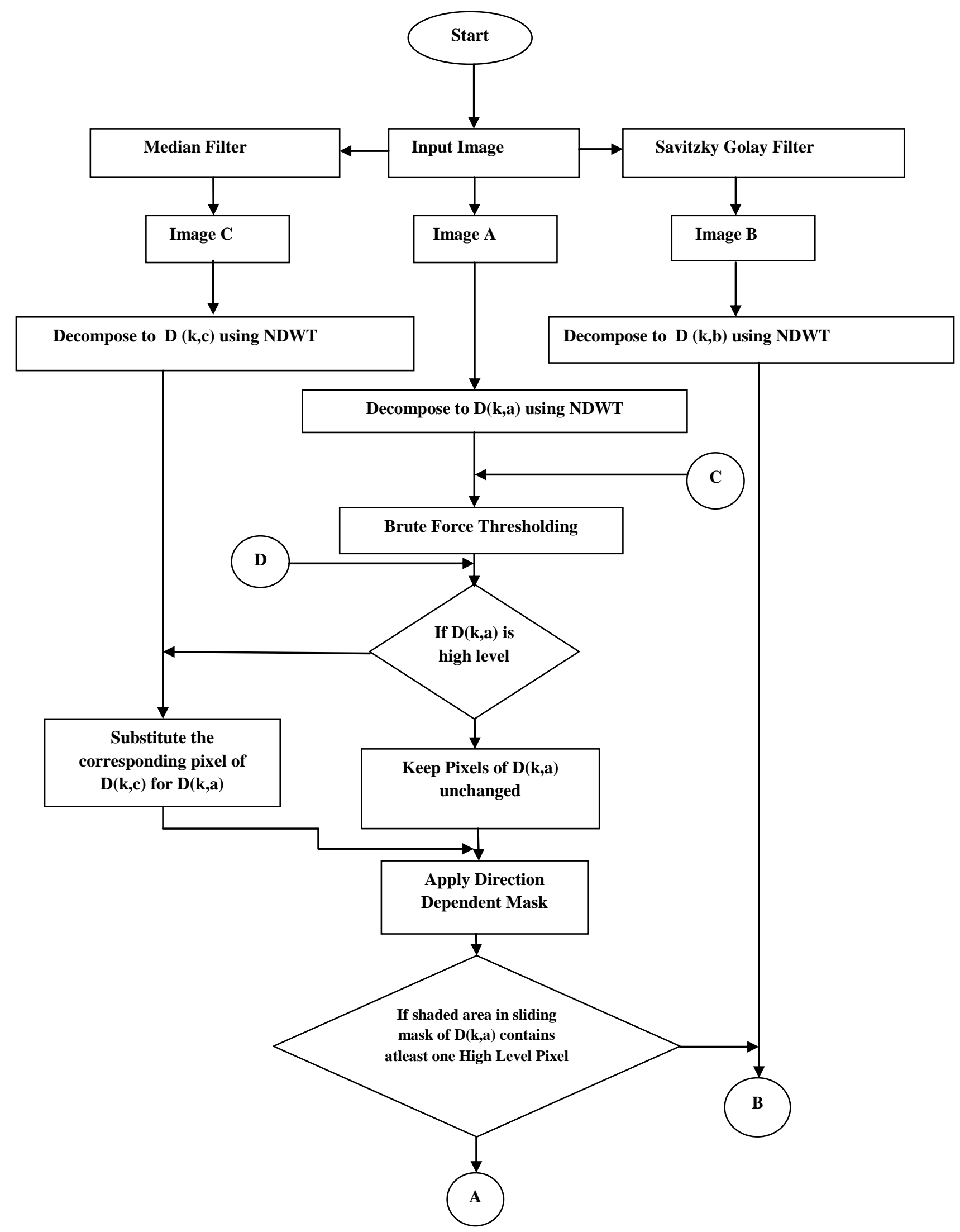




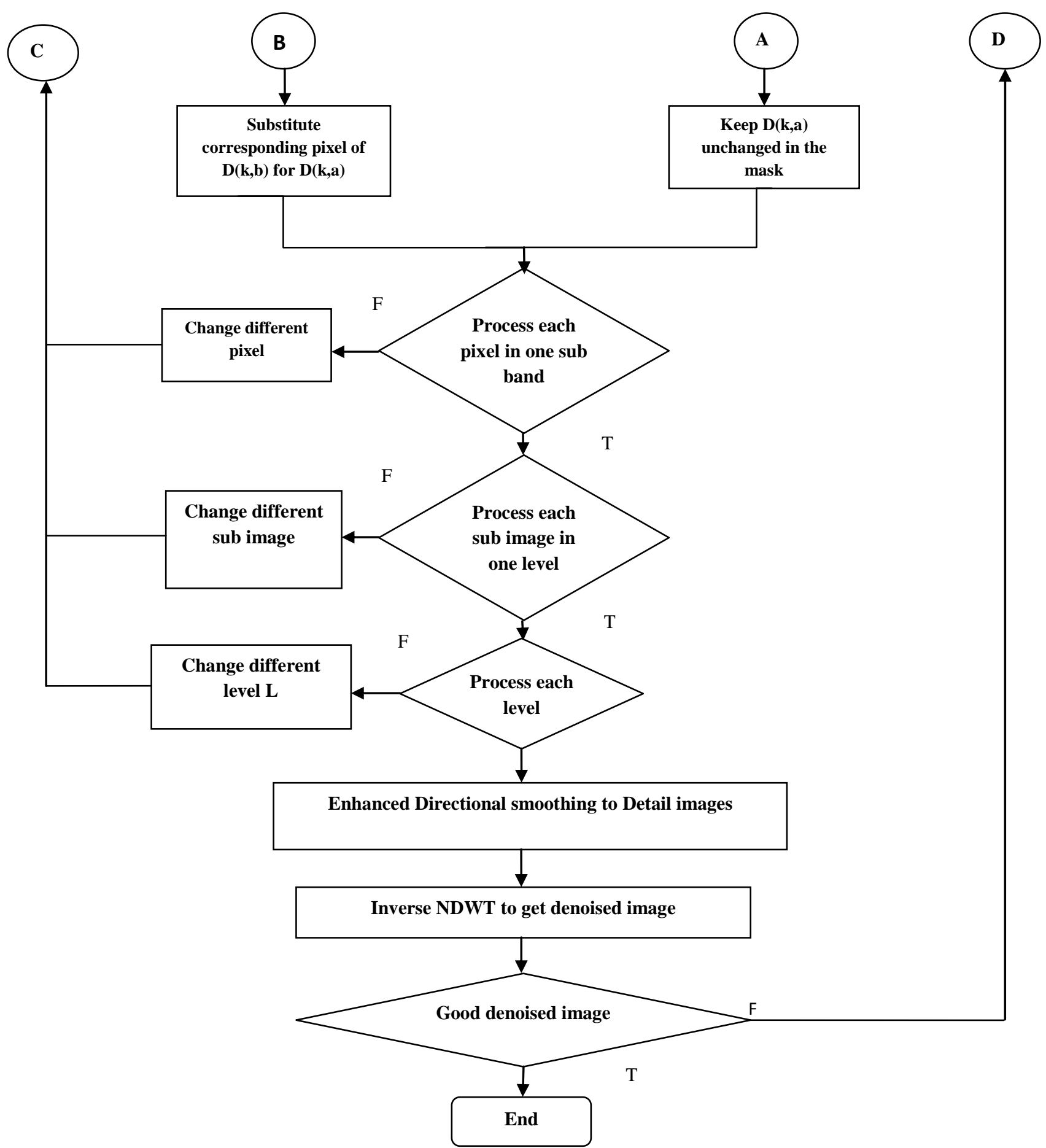

Fig.2 Detailed Flow Chart Of Proposed Algorithm.

\section{EXPERIMENTAL RESULTS}

The performance evaluation of proposed algorithm is obtained by statistical method like MSE and PSNR. The MSE should be of less value for a better filtering algorithm. The PSNR value must be high for a better filtering algorithm.

\subsection{Mean Square Error (MSE)}

MSE indicates average square difference of the pixels throughout the image between the original image (speckled) $\mathrm{g}(\mathrm{x}, \mathrm{y})$ and Despeckled image $\mathrm{f}(\mathrm{x}, \mathrm{y})$. A lower MSE means that there is a significant filter performance. But small MSE values did not always correspond to good visual quality.

$$
\mathbf{M S E}=\frac{1}{\mathbf{M N}} \boldsymbol{\Sigma} \boldsymbol{\Sigma}[\mathrm{g}(\mathrm{x}, \mathrm{y})-\mathrm{f}(\mathrm{x}, \mathrm{y})]^{2}
$$

Where $\mathrm{MxN}$ is the size of the image.

\subsection{Peak signal to Noise Ratio (PSNR)}

PSNR is used to give a quantitative evaluation. It is calculated between the original image and the noisy image. A higher PSNR would normally indicate that the reconstruction is of higher quality. PSNR is usually calculated as

$$
\text { PSNR }=10 \log _{10}\left(225^{2} / \mathrm{MSE}\right)
$$


Table 1 Comparison of proposed method with other techniques

\begin{tabular}{|l|c|c|}
\hline \multicolumn{1}{|c|}{ Technique } & PSNR & MSE \\
\hline Proposed Algorithm & 65.8148 & 0.017045 \\
\hline Wiener Filter & 61.2079 & 0.198 \\
\hline Lee Filter & 60.7267 & 1.0975 \\
\hline Mean Filter & 60.8248 & 1.073 \\
\hline Homomorphic Lee Filter & 60.4542 & 1.168 \\
\hline Frost Filter & 59.8922 & 1.330 \\
\hline Homomorphic Frost Filter & 61.7592 & 1.3714 \\
\hline Kuan Filter & 60.7317 & 1.096 \\
\hline Hard Threshold & 60.0867 & 1.097 \\
\hline Soft Threshold & 60.0245 & 1.079 \\
\hline Bayesian Threshold & 59.0558 & 1.2808 \\
\hline Normal Threshold & 59.0267 & 1.2895 \\
\hline Homomorphic Kuan Filter & 59.4639 & 1.166 \\
\hline
\end{tabular}
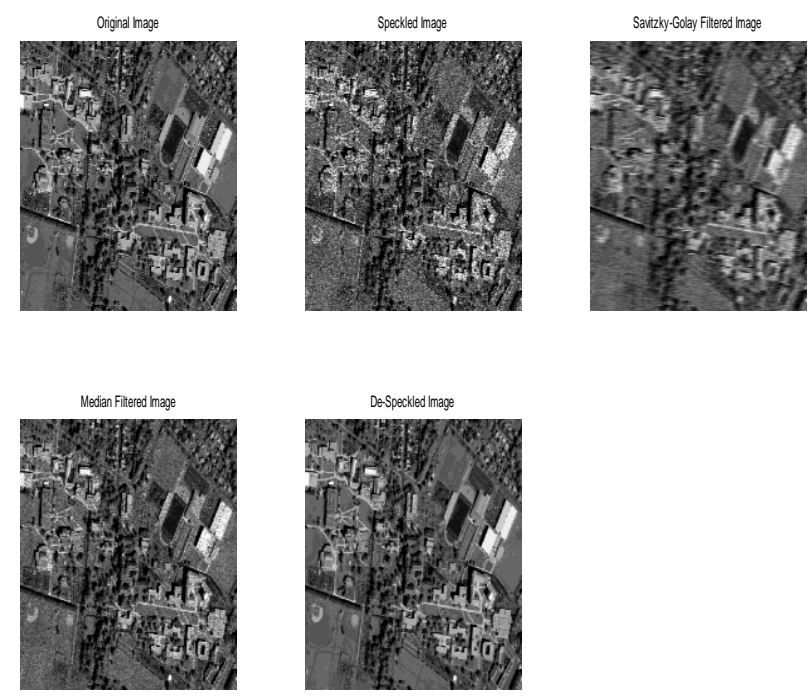

PSNR $=65.8148$ dB MSE $=0.017045$

Fig.3(a) Visual Result Of The Proposed Algorithm.
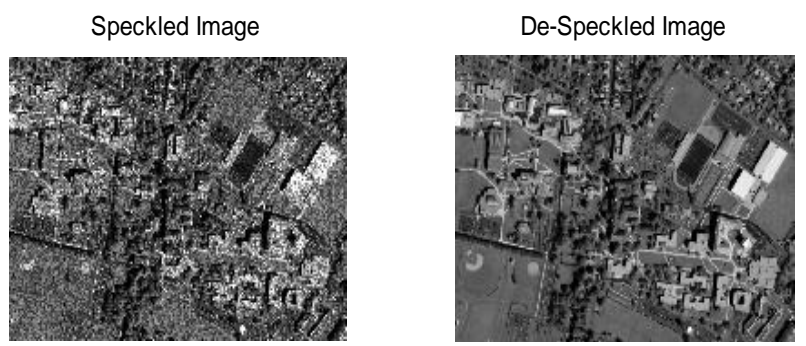

$\mathrm{PSNR}=65.8148 \mathrm{~dB}$ MSE $=0.017045$

Fig.3(b) Visual Result Of The Proposed Algorithm.

\section{CONCLUSION}

Result shows that the proposed method for despeckling is better than statistical filters and homomorphic based statistical filters. Since the desired algorithm is processed in each pixel of every level it takes more time for computation when more levels are decomposed but gives much more clear images for image processing and its analysis. With the advancement of technology, processing speed of processors increasingly rapidly, thus slightly slow execution time is no longer a big issue. The proposed algorithm always outperforms the other existing techniques but only drawback is that it is slow.

\section{REFERENCES}

[1] Guozhong Chen,Xingzhao Liu "Wavelet-Based Despeckling SAR Images Using Neighbouring Wavelet Cofficients." Proceedings of IEEE 2005.

[2] G. Lee, "Refined filtering of image noise using local statistics," Comput. Graph. Image Process., vol. 15, no. 4, 1981 .

[3] V. S. Frost, J. A. Stiles, K. S. Shanmugan, and J. C. Holtzman, "A model for radar images and its application to adaptive digital filtering of multiplicative noise," IEEE Trans. Pattern Anal. Machine Intell., vol. PAMI-4, Mar. 1980.

[4] A. Lopes, E. Nezry, R. Touzi, and H. Laur, "Maximum a posteriori filtering and first order texture models in SAR images," in Proc. IGARSS, 1990.

[5] A. Lopes, R. Touzi, and E. Nezry, "Adaptive speckle filters and scene heterogeneity," IEEE Trans. Geosci. Remote Sensing, vol. 28, pp. 992-1000, Nov. 1990.

[6] L. Gagnon and A. Jouan, "Speckle filtering of SAR images-A comparative study between complexwavelet-based and standard filters," Proc. SPIE, 1997.

[7] Birgir Bjorn Saevarsson,Johannes R.Sveinsson and Jon Atli Benediktsson "Combined Wavelet and Curvelet Denoising of SAR Images" Proceedings of IEEE 2004.

[8] Guozhong Chen,Xingzhao Liu "An Improved Waveletbased Method for SAR Images Denoising Using Data Fusion Technique". Proceedings of IEEE 2006.

[9] M.I.H.Bhuiyan,M Omair Ahmed "Wavelet-Based Spatially Adaptive Method for Despeckling SAR Images". Proceedings of IEEE 2006.

[10] Aglika Gyaourova "Undecimated Wavelet Transforms for Image Denoising" center for applied scientific computing, Lawrence Livermore nation laboratory, November 19,2002.

[11] H. Guo, J.E. Odegard, M. Lang, R.A. Gopinath, I. Selesnick, and C.S.Burrus, "Speckle reduction via wavelet shrinkage with application to SAR based ATD/R," Technical Report CML TR94-02, CML, Rice University, Houston, 1994.

[12] S.G. Chang, B. Yu, and M. Vetterli, "Adaptive wavelet thresholding for image denoising and compression," IEEE Transactions on Images Processing, vol 9, no. 9, pp.1532-1546, September 2000.

[13] X.P.Zhang, "Thresholding Neural Network for Adaptive Noise reduction," IEEE Transactions on Neural Networks, vol.12, no.3, pp567584.May 2001. 
[14] M. Amirmazlaghani and H. Amindavar, "Two Novel Bayesian Multiscale Approaches for Speckle Suppression in SAR Images," IEEE Trans. Geoscience and Remote Sensing, vol. 48, no. 7, 2010.

[15] V. Santhi,"Speckle Reduction of SAR Images using Adaptive Sigmoid Thresholding and Analysis of various Filtering Techniques", International Journal of Computer Applications, Volume 46 - Number 2, 2012.

[16] Mario Mastriani "New Wavelet-based Superresolution Algorithm for Speckle Reduction in SAR Images" IJCS volume 1 number 4, 2006.
[17] R. W. Schafer, "On the frequency-domain properties of Savitzky-Golay filter," in Proc. 2011 DSP/SPE Workshop, Sedona, AZ, Jan 2011

[18] M. Mastriani,A.E.Giraldez Enhanced Directional Smoothing Algorithm for Edge-Preserving Smoothing of Synthetic-Aperture Radar Images, SAOCOM Mission, National Commission of Space Activities (CONAE) Argentina, Feb 2004

[19] NTU satellite network, Digital Image Enhancement,10 Feb 1992 